\title{
Mitragynine (Kratom) impairs spatial learning and hippocampal synaptic transmission in rats
}

\author{
Zurina Hassan ${ }^{1 *} \mathbb{D}$, Farah W Suhaimi ${ }^{1 *}$, Surash Ramanathan ${ }^{1}$, \\ King-Hwa Ling ${ }^{2}$, Mohamad A Effendy ${ }^{1}$ (D), Christian P Müller ${ }^{3}$ \\ and Hans $C$ Dringenberg ${ }^{4}$
}

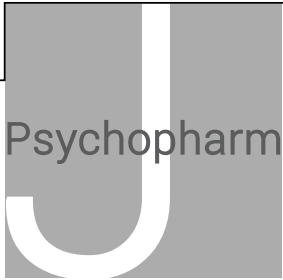

Journal of Psychopharmacology 2019, Vol. 33(7) 908-918 (c) The Author(s) 2019 Article reuse guidelines: sagepub.com/journals-permissions DOI: $10.1177 / 0269881119844186$ journals.sagepub.com/home/jop

(SAGE

\begin{abstract}
Background: Mitragynine is the major alkaloid of Mitragyna speciosa (Korth.) or Kratom, a psychoactive plant widely abused in Southeast Asia. While addictive effects of the substance are emerging, adverse cognitive effects of this drug and neuropharmacological actions are insufficiently understood. Aims: In the present study, we investigated the effects of mitragynine on spatial learning and synaptic transmission in the CA1 region of the hippocampus.

Methods: Male Sprague Dawley rats received daily (for 12 days) training sessions in the Morris water maze, with each session followed by treatment either with mitragynine $(1,5$, or $10 \mathrm{mg} / \mathrm{kg}$; intraperitoneally), morphine $(5 \mathrm{mg} / \mathrm{kg}$; intraperitoneally) or a vehicle. In the second experiment, we recorded field excitatory postsynaptic potentials in the hippocampal CA1 area in anesthetized rats and assessed the effects of mitragynine on baseline synaptic transmission, paired-pulse facilitation, and long-term potentiation. Gene expression of major memory- and addiction-related genes was investigated and the effects of mitragynine on $\mathrm{Ca}^{2+}$ influx was also examined in cultured primary neurons from E16-E18 rats.

Results/outcomes: Escape latency results indicate that animals treated with mitragynine displayed a slower rate of acquisition as compared to their control counterparts. Further, mitragynine treatment significantly reduced the amplitude of baseline (i.e. non-potentiated) field excitatory postsynaptic potentials and resulted in a minor suppression of long-term potentiation in CA1. Bdnf and $\alpha$ CaMKII mRNA expressions in the brain were not affected and $\mathrm{Ca}^{2+}$ influx elicited by glutamate application was inhibited in neurons pre-treated with mitragynine.

Conclusions/interpretation: These data suggest that high doses of mitragynine ( 5 and $10 \mathrm{mg} / \mathrm{kg}$ ) cause memory deficits, possibly via inhibition of $\mathrm{Ca}^{2+}$ influx and disruption of hippocampal synaptic transmission and long-term potentiation induction.
\end{abstract}

\section{Keywords}

Mitragynine, Kratom, Morris water maze, field excitatory postsynaptic potentials, long-term potentiation

\section{Introduction}

Kratom (or Ketum), also known as Mitragyna speciosa (Korth.), has long been used as a psychoactive plant preparation in Southeast Asia. Traditionally, the leaves of M. speciosa are reported to be used to treat intestinal infections, muscle pain, as well as to reduce coughing and diarrhea (Chuakul et al., 1995; Jansen and Prast, 1988; Said et al., 1991; Suwanlert, 1975; Vicknasingam et al., 2010; Watanabe et al., 1997). M. speciosa preparations have been used by Malay and Thai native people for their opium- and coca-like effects in order to enhance the tolerance of physical labor in high-temperature conditions (Grewal, 1932a,b; Suwanlert, 1975; Tanguay, 2011), as well as an opium substitute in the treatment of opium addiction (Ahmad and Aziz, 2012; Beckett et al., 1965; Burkill, 1935; Burkill and Haniff, 1930; Tanguay, 2011; Vicknasingam et al., 2010; Wray, 1907a,b). The main indole alkaloids present in the young leaves of $M$. speciosa are mitragynine and its analogues, speciogynine, paynantheine, and speciociliatine (Leon et al., 2009; Orio et al., 2012; Ponglux et al., 1994; Shellard et al., 1978a,b). Mitragynine constitutes $66 \%$ of the total alkaloid mixture, making it the most abundant active alkaloid derived from the leaves of $M$. speciosa (Chittrakarn et al., 2008; Jansen and Prast, 1988; Shellard, 1974, 1989; Shellard et al., 1978a,b; Takayama et al., 1998).
In humans, chronic consumption of $M$. speciosa preparations is associated with drug abuse and addiction (Hassan et al., 2013; Singh et al., 2014; Suhaimi et al., 2016). Animal studies have shown that the addiction-like effects are mediated by the main alkaloid mitragynine (Yusoff et al., 2016). Mitragynine does not only induce rewarding effects by itself (Yusoff et al., 2016; 2018), but also enhances punishment resistance in natural reward seeking (Ismail et al., 2017). Acute and chronic administrations of mitragynine also reduced locomotor activity in an open field test, impaired passive avoidance and object recognition performance (Apryani

${ }^{1}$ Centre for Drug Research, Universiti Sains Malaysia, Penang, Malaysia 2Department of Biomedical Science, Universiti Putra Malaysia,

Selangor, Malaysia

${ }^{3}$ Department of Psychiatry and Psychotherapy, Friedrich-Alexander-

University of Erlangen-Nuremberg, Erlangen, Germany

${ }^{4}$ Department of Psychology and Centre for Neuroscience Studies,

Queen's University, Kingston, ON, Canada

*These authors contributed equally.

Corresponding author:

Zurina Hassan, Centre for Drug Research, Universiti Sains Malaysia, 11800 Penang, Malaysia.

Email: zurina_hassan@usm.my 
et al., 2010; Yusoff et al., 2016). However, other investigators have not detected behavioral impairments following oral mitragynine administration in a Y-maze task designed to assess shortterm memory in mice (Hazim et al., 2011). Currently, the effects of systemic mitragynine treatment on spatial learning are unclear.

Senik et al. (2012) investigated the effects of a methanolic $M$. speciosa extract on field excitatory postsynaptic potentials (fEPSPs) and long-term potentiation (LTP) induction in the CA1 field of rat hippocampal slice preparations and reported a profound suppression of non-potentiated fEPSPs, as well as an inhibition of LTP. Further, Ilmie et al. (2015) reported that subchronic oral administration of a standardized methanolic extract of $M$. speciosa improved memory performance during the retention phase of a passive avoidance task, but inhibited LTP induction in the hippocampus CA1 region; though which compound of this extract exerted these effects remains to be determined. Given these conflicting reports and gaps in the literature, we asked whether mitragynine, the major indole alkaloid of M. speciosa, can alter spatial learning in rats, as well synaptic transmission and plasticity induction i.e. paired-pulse facilitation (PPF) and long-term potentiation in the hippocampal CA1 field under in vivo conditions. Morris water maze testing was conducted to assess spatial learning, which is correlated with hippocampal synaptic activity and plasticity. Here, morphine was used as a positive control based on two reasons. First, because mitragynine acts on the opioid receptor in a similar way to morphine (Kruegel et al., 2016; Matsumoto et al., 2014; Taufik Hidayat et al., 2010), and secondly because of its ability to affect cognitive function in rodents. Several studies have shown that morphine impairs cognition, especially with regard to learning and memory functions (Ilmie et al., 2015; Senik et al., 2012; Yusoff et al., 2016; Zhu et al., 2011). Further, we also measured basal synaptic transmission via input/output (I/O) curves and assessed short-term plasticity via PPF after treatment with mitragynine. PPF is a form of shortterm plasticity whereby the fEPSP to a second stimulus is enhanced relative to the first, if the second stimulus is delivered relatively quickly (e.g. $<500 \mathrm{~ms}$ ) after the first (Zucker, 1989). LTP is known as long-lasting, input-specific enhancement of transmission at synapses that results from concurrent, or close to concurrent, activation of both the pre- and postsynaptic neurons (Bennett, 2000; Lynch, 2004). In addition, we also wished to investigate some of the potential cellular and molecular mechanisms, especially intracellular calcium influx and the $B d n f$ and $\alpha$ CaMKII mRNA expression in the brain that could mediate alterations in hippocampal synaptic activity and plasticity induced by acute mitragynine administration.

\section{Experimental procedures}

\section{Animals}

All experimental procedures were reviewed and approved by the Institutional Animal Care and Use Committee (IACUC), Universiti Sains Malaysia (USM), Pulau Pinang, Malaysia (Approval no.: USM/Animal Ethics Approval/2013/(87)(495). Male Sprague-Dawley rats (aged 8-10 weeks) were obtained from the Animal Research and Service Centre, USM, Penang, Malaysia, weighing between 200-300 g at the time of testing. For all experiments, only new experimentally naïve animals were used. Animals were housed as groups of five rats per cage under a 12:12-hour light-dark schedule (07:00-19:00) and had free access to food and water.

\section{Mitragynine preparation and treatment}

Mitragynine was extracted, isolated, and verified from fresh leaves of M. speciosa, as described previously (Utar et al., 2011). Purified mitragynine was confirmed by high performance liquid chromatography (HPLC) and proton nuclear magnetic resonance (1H-NMR) (400 MHz) analysis (Jamil et al., 2013). Mitragynine obtained by this procedure was approximately $98 \%$ pure. For testing, a dose range of $1-10 \mathrm{mg} / \mathrm{kg}$ mitragynine was used, with detailed doses adapted for the respective experiments in pre-tests. Mitragynine was dissolved in $20 \%$ of Tween 80 as vehicle. The drug injection volume was $1 \mathrm{~mL} / \mathrm{kg}$, which was administered intraperitoneally (i.p.) immediately after completion of the daily water maze training trial for 12 days, and before the surgery for electrophysiological recording (single injection). For the Morris water maze experiments, separate groups of animals were treated with either vehicle $(n=12)$, morphine $(5 \mathrm{mg} / \mathrm{kg}, n=12)$ or mitragynine $(1,5$, or $10 \mathrm{mg} /$ $\mathrm{kg}, n=12$ /group; Yusoff et al., 2016). For electrophysiological recordings, vehicle or mitragynine ( 5 or $10 \mathrm{mg} / \mathrm{kg}$ ) were used, with doses selected based on the Morris water maze results.

\section{Morris water maze}

A circular black pool (diameter, $160 \mathrm{~cm}$ ) filled with water $\left(25 \pm 1^{\circ} \mathrm{C}\right)$ to a depth of $70 \mathrm{~cm}$ was used for water maze testing. White paint was added to the water to make the platform invisible. This maze was divided into four virtual quadrants and a stainless-steel escape platform $(10 \mathrm{~cm}$ in diameter $)$ was positioned at the center of one of these quadrants, $2 \mathrm{~cm}$ below the water surface. For each trial, an animal was lowered gently into the water, facing the pool wall at defined starting points (north, south, east, west) along the wall of the water maze. The order of the start positions was randomized.

On an initial habituation day, each animal was placed in the pool for a duration of $90 \mathrm{~s}$ in the absence of the hidden platform, to provide an estimate of swimming ability and motor control, and to familiarize rats with the test environment and some of the general task requirements (i.e. swimming). Water maze acquisition training commenced on the next day, with the hidden platform placed in the pool. Each animal was trained to navigate toward the hidden platform, with one trial per day for 12 consecutive days. An acquisition trial was terminated when the animal located and climbed onto the platform, or when $90 \mathrm{~s}$ had elapsed. In the latter case, the animal was guided and placed on the platform by the experimenter. Once the animal reached the platform, it was allowed to remain on the escape platform for 30 s. Given that rats use spatial extra-maze cues to locate the hidden platform, care was taken that the experimenter always sat at the same position during each trial and test day (Li et al., 2001). The position of the submerged platform was maintained throughout the entire training period.

Twenty-four hours after completion of the last acquisition trial, all animals were given a probe trial (drug-free) in the absence of the platform to examine the retrieval of the previously learned platform position. This probe trial lasted for $90 \mathrm{~s}$ and the time spent in each of the four pool quadrants was recorded. 
Table 1. List of primers used and their corresponding properties.

\begin{tabular}{|c|c|c|c|}
\hline Gene & Primers $\left(5^{\prime} \rightarrow 3^{\prime}\right) / \mathrm{GC} \%^{a}$ & Amplicon size (nt) & UPL probe (\#) \\
\hline$\alpha C a M K I I$ & F: AAACTGAAGGGAGCCATCCT/50 & 91 & 49 \\
\hline (alpha calcium/calmodulin-dependent protein kinase II alpha) & R: TCACGCCATCATTCTTCTTG/45 & & \\
\hline$B d n f$ & F: CGGTGGTTTATGTTGACCTGA/48 & 75 & 82 \\
\hline (brain-derived neurotrophic factor) & R: ACAGGACGGAAACAGAACGA/50 & & \\
\hline Gapdh & F: AATGTATCCGTTGTGGATCTGA/41 & 76 & 80 \\
\hline (glyceraldehyde-3-phosphate dehydrogenase) & R: GCTTCACCACCTTCTTGATGT/48 & & \\
\hline $\begin{array}{l}\text { Pgk1 } \\
\text { (phosphoglycerate kinase 1) }\end{array}$ & $\begin{array}{l}\text { F: GATGGGCTTGGACTGTGGTA/55 } \\
\text { R: AACAGGACCGTTCCAAACAA/45 }\end{array}$ & 88 & 108 \\
\hline$H m b s$ & F: GTGATGAAGGATGGGCAACT/50 & 76 & 109 \\
\hline (hydroxymethylbilane synthase gene) & R: TGGTCTCTTGCATGCTATCTG/48 & & \\
\hline
\end{tabular}

${ }^{a} \mathrm{~F}$ denotes forward primer whereas $\mathrm{R}$ denotes reverse primer.

On the next day, animals were tested for general sensorymotor performance (drug-free) in the presence of a visible platform. The trial lasted for $90 \mathrm{~s}$ or until the animal reached the platform and the time to reach the platform was recorded (de Souza Silva et al., 2013).

\section{In vivo electrophysiology}

A separate set of animals was used for the electrophysiological studies. Animals were anaesthetized with urethane $(2.0 \mathrm{~g} / \mathrm{kg}$, i.p., administered as four doses of $0.5 \mathrm{~g} / \mathrm{kg}$ each, one every $20 \mathrm{~min}$; additional $0.5 \mathrm{~g} / \mathrm{kg}$ urethane supplements were administered when necessary). After the induction of deep anesthesia, animals were mounted in the stereotaxic frame. In addition, the local analgesic Xylocaine $(5 \mathrm{mg} / \mathrm{kg}$ ) was administered subcutaneously to the skin and tissue along the incision line over the skull. Throughout the experiment, the body temperature was monitored and maintained between $36-37^{\circ} \mathrm{C}$ by an electrical heating pad and blankets covering the animal.

The skull bone was exposed and small holes were drilled over the following areas (all measurements taken from bregma and the surface of the skull): CA1: AP: $-4.2 \mathrm{~mm}, \mathrm{ML}:-3.0 \mathrm{~mm}, \mathrm{~V}:-3.0$ $\mathrm{mm}$; and CA3: AP: $-4.2 \mathrm{~mm}, \mathrm{ML}:+3.0 \mathrm{~mm}, \mathrm{~V}:-4.0 \mathrm{~mm}$. A bipolar stimulating electrode (SNE 100, MicroProbes, USA) was placed in the CA3 region to allow stimulation of the Schaffer collateral/commissural pathway (constant current pulses, $0.2 \mathrm{~ms}$ pulse duration, pulses delivered at $0.05 \mathrm{~Hz}$ ). A recording electrode (Perfluoroalkoxy (PFA) Insulated Steel Wire, A-M Systems, USA) was placed in the striatum radiatum of the CA1 region. Screws were placed in the bone overlying the frontal cortex to act as reference and ground connections for the recording electrode. The final ventral placement of the CA3 stimulating and CA1 recording electrodes was adjusted to yield maximum fEPSP amplitude in CA1 in response to CA3 stimulation.

An I/O curve was created by stimulating the CA3 field with increasing intensities $(0.1$ to $1.0 \mathrm{~mA})$ and the intensity that produced about $50-60 \%$ of the maximal fEPSP amplitude in CA1 was used for the subsequent formal data collection (PPF and LTP).

To assess short-term plasticity, PPF was measured by delivering pairs of stimulation pulses ( $0.2 \mathrm{~ms}$ duration) at interstimulus intervals (ISIs) of 20, 50, 100, 200, 500, and $1000 \mathrm{~ms}$.

To examine changes in baseline (non-potentiated) synaptic transmission, baseline (i.e. drug-free) fEPSPs were recorded for
60 min or until a stable baseline was obtained, as determined by examining fEPSPs that were averaged over successive, 10-minute recording epochs. After a stable baseline was established, drug or vehicle was administered (i.p.) and fEPSP recordings continued for three hours after drug administration.

To assess LTP, baseline recordings (same as above) of fEPSPs were followed by delivery of one theta burst stimulation (TBS) of the $\mathrm{CA} 3$ region. TBS train was composed of ten stimulus bursts delivered at $5 \mathrm{~Hz}$, with each burst consisting of five pulses at 100 $\mathrm{Hz}$. Recordings of fEPSPs continued for three hours following the delivery of TBS.

All electrophysiological signals were amplified, digitized at $10 \mathrm{kHz}$ by A PowerLab/4SP system (ADInstruments, Australia) and stored using a personal computer and LabChart v.7 software (ADInstruments) for subsequent offline analysis.

\section{Quantitative polymerase chain reaction $(q P C R)$}

In another set of experiments, rats were treated with single administration of mitragynine $(30 \mathrm{mg} / \mathrm{kg}$ i.p.). This dose was chosen because it is the highest dose that produced significant effects in our previous study (Yusoff et al., 2016). Total RNA was extracted from whole brain tissues of rats treated with $30 \mathrm{mg}$ of mitragynine and a control group ( $n=3$ per group) using Trizol reagent (Invitrogen, USA) according to the manufacturer's instructions. Extracted RNA samples passed both purity and integrity quality controls based on spectrophotometry and gel electrophoresis analysis, respectively. Subsequently, $3 \mu \mathrm{g}$ of total RNA were converted to complementary DNA (cDNA) prepared in a $20 \mu \mathrm{L}$ per reaction consisting of $10 \mathrm{mM}$ dNTP mix, $2.5 \mu \mathrm{M}$ anchored-oligo $(\mathrm{dT})_{18}, 20 \mathrm{U}$ Protector RNase Inhibitor, 1X RT buffer and $10 \mathrm{U}$ Transcriptor Reverse using the Transcriptor First Strand cDNA Synthesis Kit (Roche, USA) according to the manufacturer's instructions. Each qPCR reaction was then prepared using 0.001 $\mathrm{X}$ synthesized cDNA in a $10 \mu \mathrm{L}$ reaction consisting of $0.5 \mu \mathrm{M}$ forward and $0.5 \mu \mathrm{M}$ reverse primer, $1 \mathrm{X}$ LightCycler 480 Probes Master (Roche, USA) and $250 \mathrm{nM}$ of corresponding Universal ProbeLibrary (UPL) probes (Roche, USA) (see Table 1). All qPCR assays were designed by using UPL Assay Design Centre (www.universalprobelibrary.com) and validated with PCR efficiencies between $98-101 \%$ with $\mathrm{R}^{2}>0.990$. The validation step was performed on a serially diluted pooled cDNA synthesized 
from the samples described above. All qPCR reactions were cycled in LightCycler 480 Real-Time PCR System using the 96-well plate format. All qPCR reactions were pre-denatured at $95^{\circ} \mathrm{C}$ for $10 \mathrm{~min}$, followed by 45 -cycle of $95^{\circ} \mathrm{C}$ for $10 \mathrm{~s}$ (denaturation), $60^{\circ} \mathrm{C}$ for $30 \mathrm{~s}$ (annealing and elongation) and $72^{\circ} \mathrm{C}$ for $1 \mathrm{~s}$ (signal detection). Fluorescence signals were detected based using the $465 \mathrm{~nm}$ and $510 \mathrm{~nm}$ excitation and emission filters, respectively. Cycle threshold $(\mathrm{Ct})$ values were determined based on the second derivative maximum method (Luu et al., 2005) and the differences of expression levels between groups were analyzed based on the relative quantification by standard curve approach. Intrasample normalization of brain-derived neurotrophic factor $(B d n f)$ and alpha calcium/calmodulin-dependent protein kinase II $(\alpha C a M K I I)$ (Müller et al., 2016) genes against all the three housekeeping genes, Gapdh, Pgk1, and Hmbs were adopted to calculate the geometric means for statistical testing.

\section{Rat primary neuron culture}

Rat embryonic day 16 (E16) to E18 cortical neurons were cultured on glass coverslips coated with poly-D-lysine in Neurobasal medium with B27 supplement (Invitrogen) containing $100 \mathrm{IU} /$ $\mathrm{mL}$ penicillin and $100 \mu \mathrm{g} / \mathrm{mL}$ streptomycin. Twenty-four hours after plating, cultures were treated with $1 \mu \mathrm{M}$ cytarabine for $48 \mathrm{~h}$ to remove contaminating glia. Cells were used at 14-16 days for all calcium imaging experiments.

\section{Fura-2 calcium imaging}

Intracellular calcium measurement has been described previously (Damodaran et al., 2018). In brief, primary cultured neurons were incubated in the dark for 30 min with $2.5 \mu \mathrm{M}$ fura-2 acetoxymethyl (Fura-2 AM) (Thermo Fisher Scientific). After being gently washed with normal bath solution containing 140 $\mathrm{mM} \mathrm{NaCl}, 5 \mathrm{mM} \mathrm{KCl}, 1.8 \mathrm{mM} \mathrm{CaCl} 2.2 \mathrm{H} 2 \mathrm{O}, 1 \mathrm{mM}$ $\mathrm{MgCl} 2.6 \mathrm{H} 2 \mathrm{O}, 10 \mathrm{mM}$ HEPES, $10 \mathrm{mM}$ D-Glucose (pH 7.3), the cells were placed on the Olympus IX81 microscope with the MT-20 illumination unit. Fluorescence was recorded at excitation wavelengths of $340 \mathrm{~nm}$ and $380 \mathrm{~nm}$ with $300 \mathrm{~ms}$ intervals. The Fura-2 ratio of F340/F380 was calculated as an indicator of intracellular $\left[\mathrm{Ca}^{2+}\right]_{\mathrm{i}}$. Mitragynine was first dissolved in $100 \%$ dimethylsulfoxide (DMSO) to yield a $10 \mathrm{mM}$ solution, and then diluted in bath solution. The neurons were incubated with or without mitragynine for $2.5 \mathrm{~min}$ before being bathed in glutamate solution $(2 \mu \mathrm{M}$ L-glutamic acid, $140 \mathrm{mM} \mathrm{NaCl}, 5 \mathrm{mM}$ $\mathrm{KCl}, 1 \mathrm{mM} \mathrm{MgCl} 2,1.8 \mathrm{mM} \mathrm{CaCl} 2,10 \mathrm{mM}$ HEPES, $10 \mathrm{mM}$ glucose) for 30 seconds. The data were analyzed using Xcellence Rt software (Olympus).

\section{Statistical analysis}

All data were expressed as mean \pm standard error of the mean (SEM) using Sigma Plot 13.0. Generally, data were analyzed using analysis of variance (ANOVA), followed by Bonferroni post-hoc tests. For the electrophysiological data, the maximum amplitude of the fEPSP was computed offline using LabChart v.7 software (ADInstruments). Amplitude data $(\mathrm{mV})$ were averaged over 10-minute intervals and then normalized by dividing each value by the average baseline (pre-TBS) amplitude of the animal.
For qPCR analysis, the differences between the two groups were tested by using unpaired $t$ test with Welch's correction (no assumption of equal standard deviation). The statistical differences were significant at $p<0.05$.

\section{Results}

\section{Mitragynine impairs spatial learning and reference memory in the Morris water maze}

Repeated exposure to mitragynine for 12 days (administered each day after completion of the daily training trial) resulted in an impairment in acquisition of an escape response in the Morris water maze task (Figure 1(a)). The escape latencies were significantly prolonged in morphine $(5 \mathrm{mg} / \mathrm{kg})$ and mitragynine-treated animals ( 5 and $10 \mathrm{mg} / \mathrm{kg}$ ) when compared to the vehicle-treated animals. For morphine-treated animals, escape latency was significantly prolonged on day 5 to day $7(p<0.05)$. As for the mitragynine-treated groups, the escape latency was remarkably prolonged in animals that received mitragynine doses 5 and $10 \mathrm{mg} / \mathrm{kg}$. Significant increases in escape latencies were observed on training days 4 to 8 . Towards the end of the training trial (day 10-12), all groups reached an asymptotic performance level. However, at the low dose of mitragynine (1 mg/ $\mathrm{kg}$ ), water maze performance did not differ between drug- and vehicle-treated animals $(p>0.05)$. A two-way repeated measure ANOVA conducted on the escape latency data confirmed significant differences due to treatment $\left(F_{4,51}=4.430 ; p=0.004\right)$ and training days $\left(F_{11,561}=36.380 ; p<0.0001\right)$. However, there was no significant interaction between training days and treatment $\left(F_{44,561}=1.22 ; p=0.160\right)$.

Twenty-four hours after the last acquisition trial, all animals were subjected to probe trial (drug-free) testing in order to determine the time spent in the target quadrant (no platform). The time spent in the target quadrant was significantly reduced for animals that had received mitragynine at doses of $5 \mathrm{mg} / \mathrm{kg}(p=0.015)$ and $10 \mathrm{mg} / \mathrm{kg}(p=0.0049)$ during the previous 12 training days (Figure 1(b)). However, at $1 \mathrm{mg} / \mathrm{kg}$ mitragynine, probe trial performance was not impaired compared to rats that had received vehicle treatment during training. In contrast to the effect of mitragynine, animals in the morphine-treated group displayed intact probe-trial performance. It is important to note that drug treatments did not exert any long-term effects on general sensory motor performance, as evidenced by a lack of differences in the escape latencies when animals were required to swim to a visible platform $24 \mathrm{~h}$ after the probe trial test (Figure 1(c)).

\section{Mitragynine suppresses non-potentiated fEPSPs in the hippocampal CA1 region}

The effect of mitragynine on baseline (non-potentiated) transmission at CA3-CA1 synapses was investigated. For both vehicleand mitragynine-treated animals $(5 \mathrm{mg} / \mathrm{kg}$; injections given after $60 \mathrm{~min}$ of drug-free baseline fEPSP recordings), fEPSP amplitude remained relative stable throughout the three-hour period following drug administration (amplitude values in the range of $89-104 \%$ of baseline; Figure 2). However, in rats given the high dose $(10 \mathrm{mg} / \mathrm{kg})$ of mitragynine, fEPSP amplitude showed a marked decline to about $54 \%$ of baseline values, an effect that 


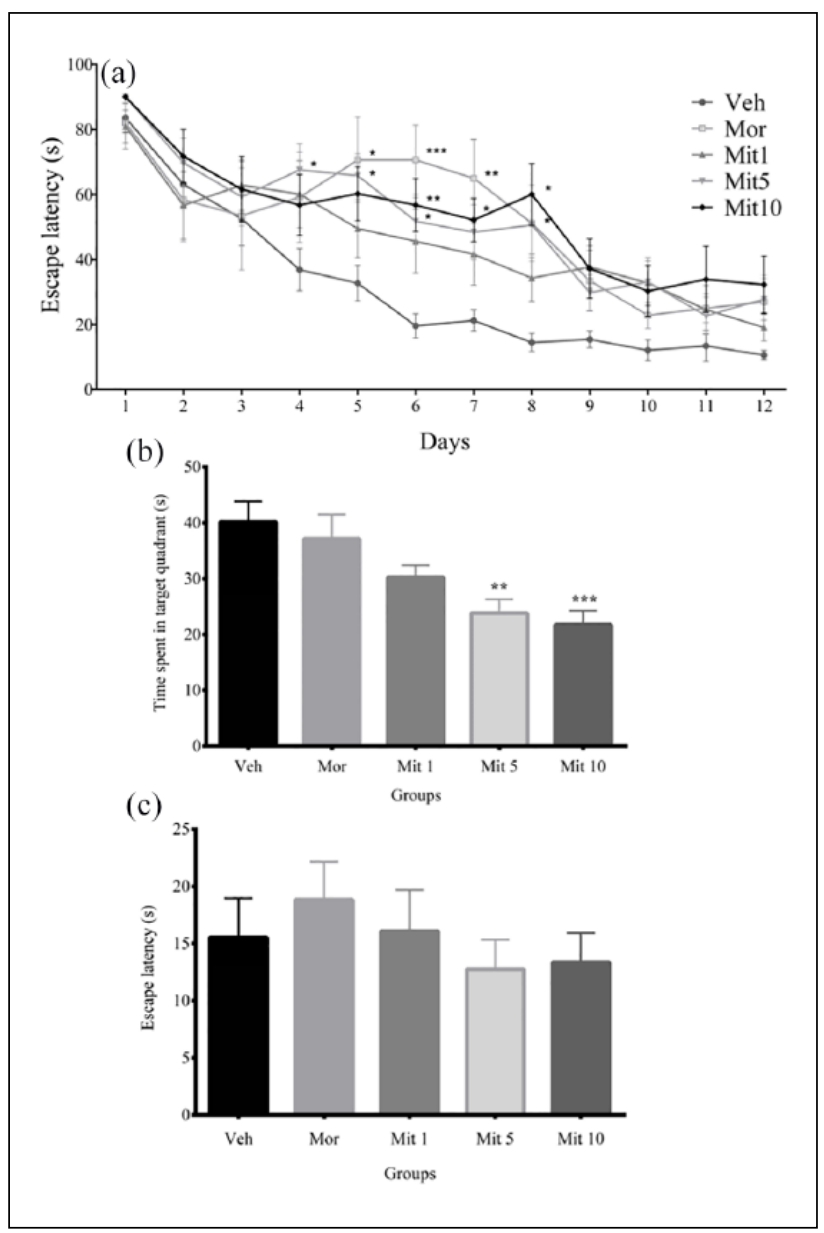

Figure 1. The effects of vehicle (Veh), mitragynine (Mit; 1, 5, and $10 \mathrm{mg} / \mathrm{kg}$ ), or morphine (Mor; $5 \mathrm{mg} / \mathrm{kg}$ ) on performance in the Morris water maze. (a) Escape latencies (mean \pm standard error of the mean (SEM)) during 12 consecutive training days (one training trial/day). (b) Time spent in the target quadrant during probe trial testing. (c) Escape latencies for the visible platform test $\left({ }^{*} p<0.05,{ }^{* *} p<0.01,{ }^{* * *} p<0.001\right.$, vs the vehicle-treated group; $n=12$ / for all groups).

emerged from the 100th minute to $240 \mathrm{~min}$ of the experiment. A two-way ANOVA showed an effect of mitragynine on non-potentiated fEPSP amplitude in the CA1 region, with a significant effect of treatment $\left(F_{2,216}=92.90, p<0.0001\right)$ and time $\left(F_{23,216=} 5.214, p<0.0001\right)$, as well as a treatment $\times$ time interaction $\left(F_{46,216}=2.184, p<0.0001\right)$.

These results were confirmed in a separate analysis assessing the effects of mitragynine on fEPSPs recorded during the I/O generation (single $0.2 \mathrm{~ms}$ pulses delivered to $\mathrm{CA} 3$ at intensities between $0.1-1.0 \mathrm{~mA}$ in $0.1 \mathrm{~mA}$ increments). Results showed that mitragynine $(10 \mathrm{mg} / \mathrm{kg})$ significantly altered I/O curves (Figure $3(a)$ ), with a two-way repeated measure ANOVA revealing significant effects of stimulation intensity $\left(F_{9,108=} 190.266, p=0.0001\right)$ and drug treatment $\left(F_{2,12}=4.537, p=0.034\right)$, as well as an intensity by drug interaction $\left(F_{18,108}=2.462, p=0.0022\right)$. The fEPSP amplitude was significantly reduced at the $0.3,0.4,0.5$, and $0.6 \mathrm{~mA}$ stimulation intensities in animals treated with mitragynine 10 $\mathrm{mg} / \mathrm{kg}$ in comparison to vehicle-treated rats (Figure 3(a)).

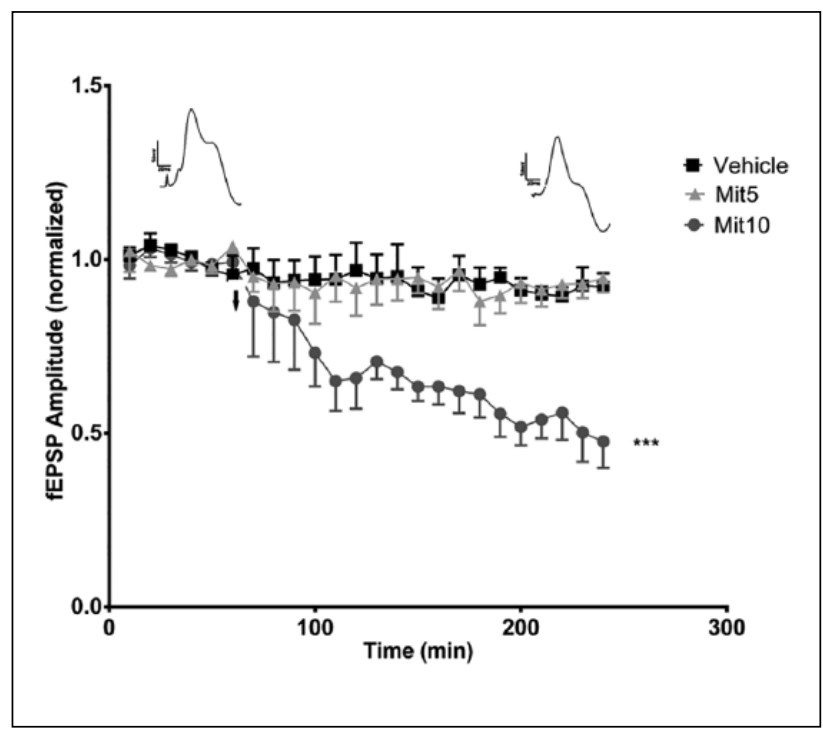

Figure 2. The effect of vehicle and mitragynine (Mit; 5 and $10 \mathrm{mg} / \mathrm{kg}$; $n=4$ for all groups) in the absence of theta burst stimulation to assess changes in baseline (non-potentiated) synaptic strength in the hippocampus CA1 region following drug treatment. Stable baseline field excitatory postsynaptic potentials (fEPSPs) were recorded for $60 \mathrm{~min}$, followed by drug or vehicle administration (intraperitoneally (i.p.); at arrow). Vehicle or a low dose $(5 \mathrm{mg} / \mathrm{kg})$ of mitragynine did not result in significant changes in fEPSP amplitude. However, the high dose $(10 \mathrm{mg} / \mathrm{kg})$ of mitragynine produced a significant suppression of fEPSPs over the three hours following drug administration. Insets: traces of fEPSPs recorded during baseline and at $240 \mathrm{~min})$. The data are expressed as the mean \pm standard error of the mean (SEM). ${ }^{* * *} p<0.001$ vs the vehicle-treated group at $240 \mathrm{~min}$.

\section{Effects of mitragynine on hippocampal PPF and long-term potentiation}

The effect of mitragynine on PPF in the hippocampal CA1 region was investigated. Treatment groups received mitragynine (i.p.; either 5 or $10 \mathrm{mg} / \mathrm{kg}$ ) and control animals were given $20 \%$ Tween 80 (vehicle). In controls, PPF was observed for paired pulses delivered at ISIs between 20-200 ms (Figure 3(b)). Mitragynine did not significantly affect PPF when compared to the control group, with no significant difference between treatment groups $\left(F_{2,12}=1.313, p=0.3051\right)$ and no treatment by ISI interaction $\left(F_{10,60}=1.047, p=0.4168\right)$. However, there was a significant effect of ISI $\left(F_{5,60}=5.112, p=0.0006\right)$, confirming that shorter ISIs were more effective than longer ones in eliciting PPF (Figure 3(b)).

Next, we assessed the effects of mitragynine ( 5 and $10 \mathrm{mg} / \mathrm{kg}$ ) on LTP in CA1 induced by TBS applied to CA3. In control animals $(20 \%$ of Tween 80$)$, the TBS produced sustained LTP, with maximum potentiation of over $100 \%$ potentiation and lasting till the end of the experiment (about $60 \%$ potentiation at three hours after TBS delivery; Figure 3(c)). Treatment with $10 \mathrm{mg} / \mathrm{kg}$ mitragynine resulted in fEPSP amplitude is comparable to that in control animals (Figure 3(c)). However, for animals treated with mitragynine at $5 \mathrm{mg} / \mathrm{kg}$, maximal potentiation reached only about $80 \%$ and declined to about $40 \%$ at the end of the experiment. Further, levels of potentiation in rats given $5 \mathrm{mg} / \mathrm{kg}$ mitragynine 


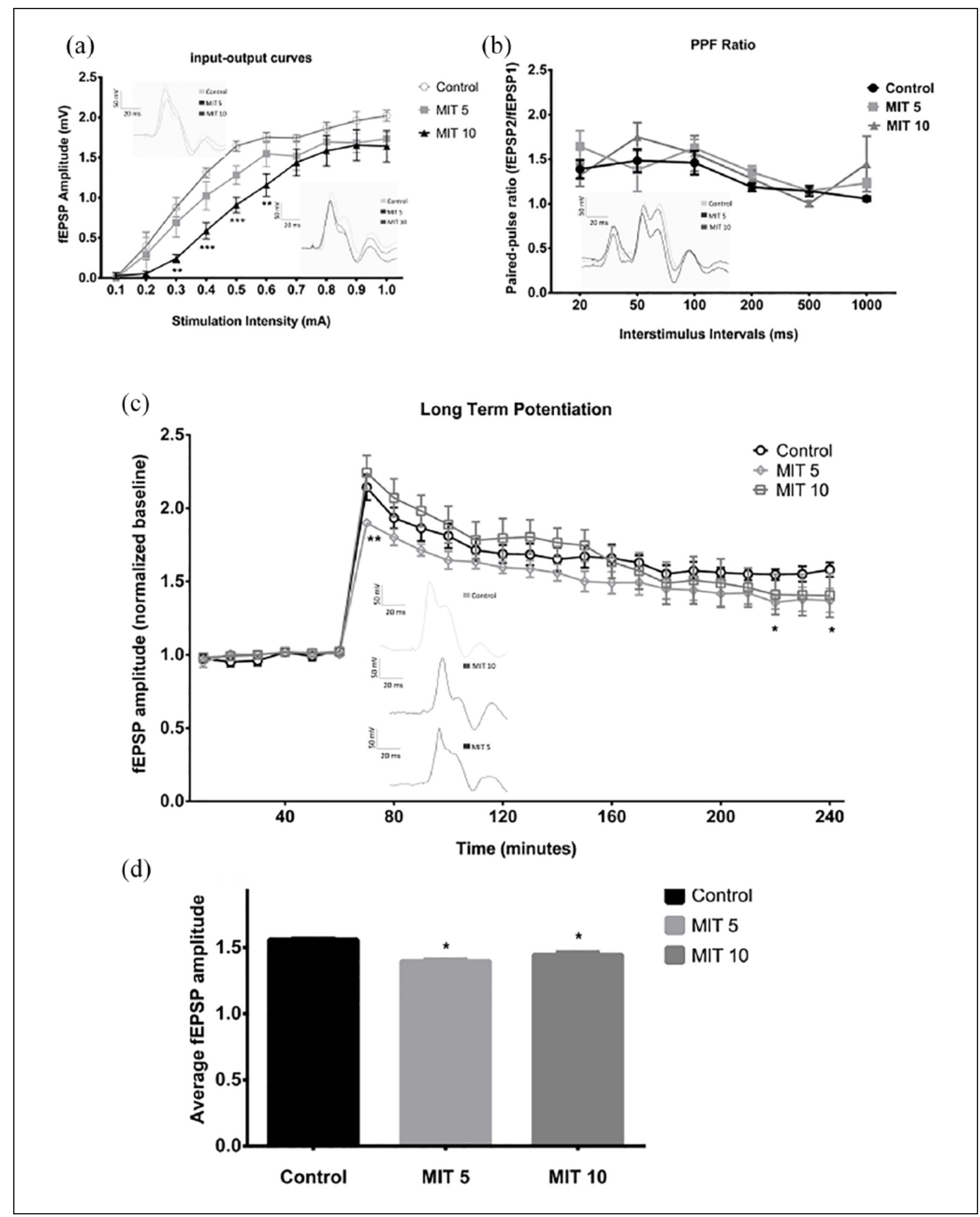

Figure 3. Effects of vehicle (Control) and mitragynine (Mit 5 and $10 \mathrm{mg} / \mathrm{kg}$ ) administration on field excitatory postsynaptic potentials (fEPSPs) in the hippocampal CA1 region of urethane-anesthetized rats (data are expressed as the mean \pm standard error of the mean (SEM) ( $n=5 / \mathrm{group}$ ). (a) Input-output (I/0) curves generated with increasing stimulation intensities (0.1-1.0 mA) delivered to the contralateral CA3 field. Mitragynine administration $(10 \mathrm{mg} / \mathrm{kg}$ ) resulted in reduced fEPSP amplitude at stimulation intensities between 0.3-0.6 mA. Inserts depict typical fEPSPs elicited by 0.5 and $1.0 \mathrm{~mA}$ in rats treated with vehicle (larger amplitude) and $10 \mathrm{mg} / \mathrm{kg}$ mitragynine (smaller amplitude) $\left({ }^{* *} p<0.01,{ }^{* *} p<0.001\right.$, vs the vehicle-treated group). (b) Paired-pulse facilitation (PPF) was assessed by applying pairs of stimuli to CA3 at interstimulus intervals ranging from 20-1000 ms and calculating the amplitude ratio of the two fEPSPs elicited in CA1. There were no significant differences among the various treatment groups. Inserts depict typical fEPSPs elicited at an interstimulus interval of 20 ms. (c) Long-term potentiation (LTP) was assessed by recording stable baseline fEPSPs for $60 \mathrm{~min}$, followed by theta burst stimulation (TBS) delivery (immediate after 60 min). Mitragynine administration $(5 \mathrm{mg} / \mathrm{kg})$ resulted in a modest suppression of LTP $\left({ }^{*} p<0.05\right.$ between Mit 5 and Control). Inserts depict typical fEPSPs recorded at $240 \mathrm{~min}$ in a control animal (top) and in rats treated with either Mit 5 (middle) or Mit 10 (bottom). (d) Average fEPSP amplitudes recorded from 121-240 min of the experiment in the three treatment conditions ( ${ }^{*} p<0.05$ compared to Control). 
and vehicle differed significantly at 70, 220, and $240 \mathrm{~min}$ of the experiment (Figure 3(c)). Two-way repeated measure ANOVA revealed a significant time effect $\left(F_{23,276}=104.665, p<0.0001\right)$, but no effect of treatment effect $\left(F_{2,12}=1.095, p=0.3657\right)$. However, there was a significant time $\times$ treatment interaction

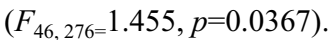

In an additional analysis, mean values of the fEPSP amplitude (normalized to pre-TBS baselines) for the last two hours of the experiment (i.e. from two to three hours following LTP induction) were compared among the three experimental groups (Figure 3(d)). This analysis showed that, compared to vehicletreated rats (fEPSP amplitude at $1.68+0.03$ of baseline), LTP levels were significantly lower in mitragynine-treated animals at both the $5 \mathrm{mg} / \mathrm{kg}(1.49+0.04 ; p<0.05)$ and $10 \mathrm{mg} / \mathrm{kg}$ dose $(1.48+0.08 ; p<0.05)$.

\section{Effects of mitragynine on expression levels of Bdnf and $\alpha$ CaMKII}

Quantitative analysis of $B d n f$ (adjusted $p$ value $=0.3943$ ) and $\alpha C a M K I I$ (adjusted $p$ value $=0.8416$ ) gene expression levels showed no significant differences between mitragynine-treated animals and the control groups (Figure 4), suggesting that drug treatment did not alter the gene expression profile for $B d n f$ and $\alpha C a M K I I$ in the whole brain samples.

\section{Effects of mitragynine on $\mathrm{Ca}^{2+}$ influx}

To examine the effects of mitragynine on $\mathrm{Ca}^{2+}$ influx, we cultured primary neurons from E16-E18 rats. The neurons received vehicle DMSO or various concentrations of mitragynine treatments for around three minutes, followed by $30 \mathrm{~s}$ of $2 \mu \mathrm{M}$ glutamate. The results from two sample neurons are shown in Figure 5(a) and (b). Treatment of vehicle and mitragynine at various concentrations did not alter $\left[\mathrm{Ca}^{2+}\right]_{\mathrm{i}}$. However, pre-treatment with mitragynine at all three concentrations $(10,30$, and $100 \mu \mathrm{M})$ inhibited $\mathrm{Ca}^{2+}$ influx upon glutamate stimulation as compared to the vehicle group (Figure 5(c)). This result demonstrates that mitragynine may impair calcium homeostasis via inhibiting $\mathrm{Ca}^{2+}$ influx through glutamate receptors.

\section{Discussion}

Kratom is a widely abused drug in Southeast Asia with largely unknown impacts on the cognitive performance of users. Here, we investigated the effects of its main psychoactive ingredient, mitragynine, on spatial learning using the Morris water maze and hippocampal synaptic transmission and plasticity. The water maze task has been extensively used as a tool to evaluate the effects of drugs on spatial learning, which often correlates with activity and plasticity in the septo-hippocampal system (Zheng et al., 2002). In the present study, daily post-training exposure to mitragynine impaired the acquisition of spatial learning in the Morris water maze, as well as memory retrieval during the probe trial. This deficit in spatial learning closely resembles the effects of morphine noted in the present and other studies (McNamara and Skelton, 1992). Interestingly, memory assessed during the probe trial showed that morphine-treated animals were still able to recognize the quadrant where the escape platform was previously placed, while mitragynine treatment ( 5 and $10 \mathrm{mg} / \mathrm{kg}$ ) significantly impaired probe trial performance. Thus, mitragynine appears to produce more severe and long-lasting disruptions of spatial memory formation than those associated with morphine administration. Numerous previous studies have also shown that mitragynine at the dose range tested here does not impair locomotor performance or coordination (Apryani et al., 2010; Yusoff et al., 2016). Thus, learning deficits concluded from behavioral performance in the Morris water maze may not have resulted from impaired swimming performance. In our study, we used the cued learning trial (Vorhees and Williams, 2006) to detect the presence of unspecific factors (i.e. sensory-motor deficits) that might affect the animals' cognitive performance. The cued learning trial showed no significant difference in escape latencies between groups in the presence of the visible platform, which indicates that sensory-motor functions were not compromised in the drug-treated groups (Figure 1(c)).

A study by Zhu et al. (2011) showed that morphine treatment impaired both spatial learning and reference memory. Some studies suggest that spatial working memory during acquisition may not be a crucial step towards forming a reference memory (Bannerman et al., 2008; Niewoehner et al., 2007), which may explain the ability of morphine-treated rats to perform well on the probe trial (present study results). These findings also support the notion that different mechanisms may underlie spatial learning and reference memory formation (Izquierdo et al., 2000).

This is the first report that investigated the effects of mitragynine on baseline (non-potentiated) transmission in the hippocampal CA1 region using fEPSP recordings in vivo. We found that after vehicle and low dose mitragynine $(5 \mathrm{mg} / \mathrm{kg})$ applications, fEPSP amplitude remained relative consistent throughout the three-hour period following drug treatment. However, mitragynine $(10 \mathrm{mg} / \mathrm{kg})$ induced a significant synaptic depression by reducing the fEPSP amplitude to about $54 \%$ of baseline values. Thus, it appears that relatively high doses of mitragynine interfere with the hippocampal synaptic transmission by depressing glutamatergic signaling in CA1 neurons (Simon et al., 2001). This conclusion was further supported by analyzing (I/O) curves for fEPSPs elicited by increasing stimulation intensities applied to the contralateral CA3 field (Dunlop et al., 2008). Compared to vehicle-treated animals, I/O curves obtained in the mitragynine group $(10 \mathrm{mg} / \mathrm{kg})$ showed a significantly smaller fEPSP amplitude at the stimulation intensity between $0.3-0.6 \mathrm{~mA}$ (Figure 3(a)), confirming the effect of mitragynine of disrupting hippocampal synaptic transmission.

PPF is a form of short-term synaptic plasticity elicited by two stimulation pulses delivered at a short ISI (typically $<500 \mathrm{~ms}$ ). At least in part, PPF is thought to be related to increases in transmitter release from presynaptic terminals as a result of $\mathrm{Ca}^{2}$ accumulation following the rapid application of stimulation pulses (Thomson, 2000). Mitragynine application did not result in any significant changes in PPF under the present experimental conditions. Thus, it appears that, in contrast to the pronounced effects of basal synaptic transmission, mitragynine does not exert major effects on this type of short-term synaptic plasticity in the hippocampal CA1 field.

For all electrophysiological experiments, vehicle and mitragynine ( 5 or $10 \mathrm{mg} / \mathrm{kg}$ ) were given to the rats i.p. 15-30 min before the start of urethane anesthesia to allow the drug to reach its maximum concentration $\left(\mathrm{C}_{\max }\right)$ during the electrophysiological 


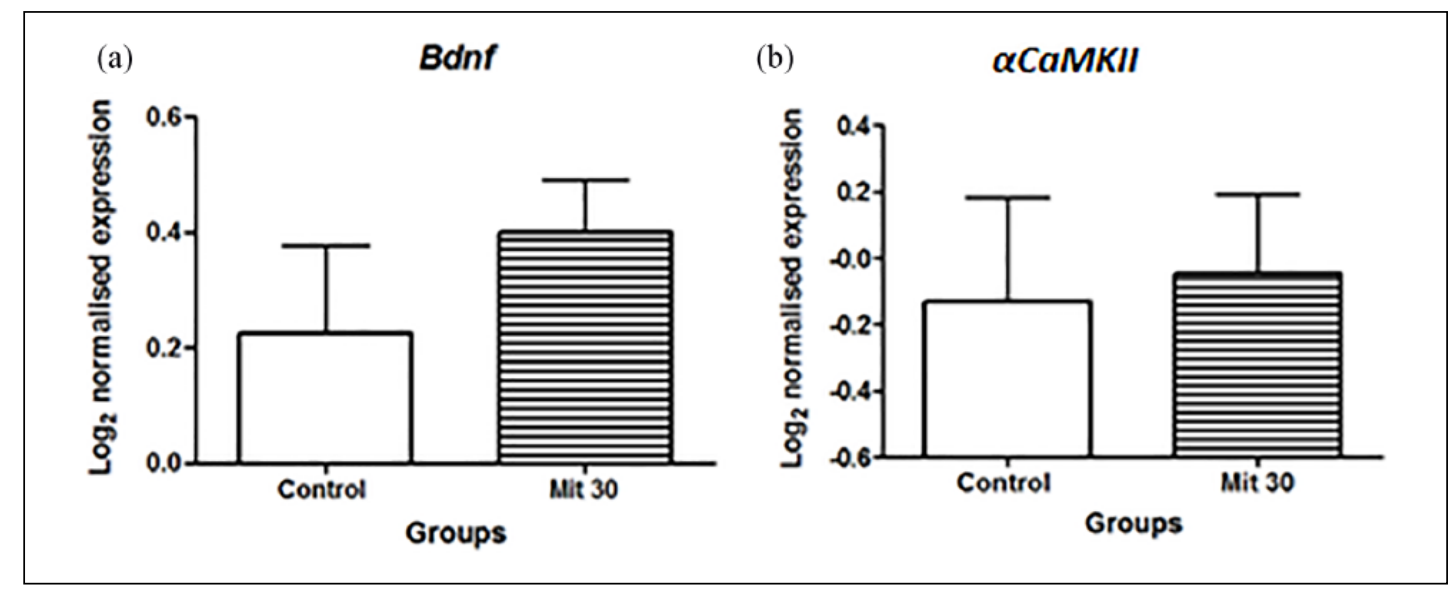

Figure 4. (a) Brain-derived neurotrophic factor (Bdnf) and (b) alpha calcium-calmodulin-dependent protein kinase II ( $\alpha$ CaMKII) gene expression levels in mitragynine-treated (Mit; $30 \mathrm{mg} / \mathrm{kg}$ ) and control rat whole-brain samples. The data are expressed as the mean \pm standard error of the mean (SEM) ( $n=3 /$ group).

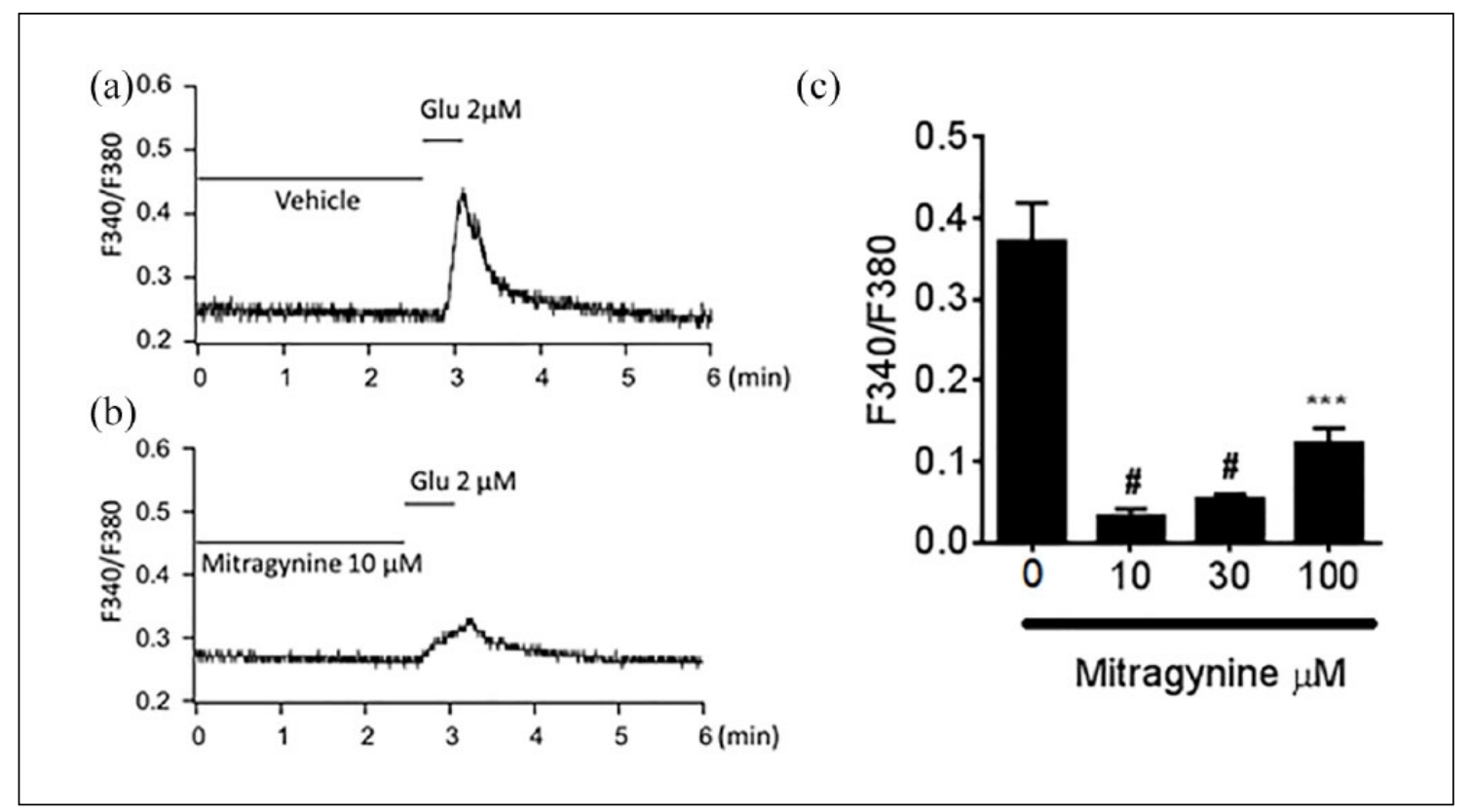

Figure 5. $\mathrm{Ca}^{2+}$ influx elicited by glutamate (Glu) is inhibited in neurons pretreated with mitragynine. (a) Graph of the Fura-2 ratio of a sample neuron treated with vehicle (dimethylsulfoxide (DMSO)) and $2 \mu \mathrm{M}$ Glu. (b) Graph of the Fura-2 ratio of a sample neuron treated with $10 \mu \mathrm{M}$ mitragynine, followed by $2 \mu \mathrm{M}$ Glu. (c) Summary of the Fura-2 ratio changes in neurons receiving Glu application following pre-treatment of either vehicle (DMSO) or various concentrations of mitragynine. ${ }^{* *} p<0.001$ (vs DMSO), $\# p<0.0001$ (vs DMSO), one-way analysis of variance (ANOVA) followed by Bonferroni post-hoc test.

recordings. The drug was expected to reach its maximum concentration in the body within an hour post-administration. In the literature, there is only a small number of studies on mitragynine pharmacokinetics in rats after oral administration. In most cases, the drug was found to reach $\mathrm{C}_{\max }$ about $1-2 \mathrm{~h}$ after oral administration (20,40, $50 \mathrm{mg} / \mathrm{kg}$ mitragynine) (de Moraes et al., 2009; Janchawee et al., 2007; Parthasarathy et al., 2010). In some instances, perhaps due to its erratic absorption, the plasma concentration over time did not clearly indicate a single time-point of $\mathrm{C}_{\max }$, but the drug apparently peaked at one and five hours with approximately similar concentrations, and the declined gradually over time (Parthasarathy et al., 2010). In our laboratory, we found that the drug reached a peak at one hour post-administration at $\sim 170 \mathrm{ng} / \mathrm{mL}$ (plasma level) after a single oral dose of $20 \mathrm{mg} / \mathrm{kg}$ mitragynine. This is in line with its physicochemical properties, given that mitragynine is lipophilic in nature, acid labile, and of poor water solubility (Ramanathan et al., 2015). In rats, oral administration of this drug resulted in very poor bioavailability (3\%) (Parthasarathy et al., 2010). However, its intermediate lipophilicity favored rapid entrance and elimination from inner tissue compartments after intravenous administration. Also, mitragynine, being a psychotropic drug, is expected to show rapid blood 
brain barrier (BBB) penetration. Based on the current studies from our laboratory, mitragynine has shown a moderate capacity to accumulate inside brain parenchymal cells (Yusof et al., 2018). In recognition of poor bioavailability following oral administration, an intraperitoneal route of administration was used, which should have resulted in improved bioavailability and a shorter delay to reach $\mathrm{C}_{\max }$ in plasma.

To analyze the effects of mitragynine on long-term potentiation, we used TBS in the CA3 field to induce LTP of fEPSPs in the hippocampal CA1 area. In control animals, TBS produced over $100 \%$ maximal potentiation of fEPSP amplitude, which declined by approximately $30 \%$ by the end of the experiment. Animals treated with mitragynine at $10 \mathrm{mg} / \mathrm{kg}$ showed levels of LTP that were similar to those seen in the control group. However, there was a statistically significant reduction in fEPSP amplitude averaged over the last two hours of the experiment (i.e. from 61 to $180 \mathrm{~min}$ after LTP induction) in rats given $10 \mathrm{mg} / \mathrm{kg}$ mitragynine compared to vehicle-treated animals. In the group which received the $5 \mathrm{mg} / \mathrm{kg}$ dose, maximal fEPSP amplitude after TBS reached only $70 \%$ and LTP gradually declined to about $40 \%$ by the end of the experiment. These results suggest that mitragynine may impair long-term synaptic plasticity in the hippocampal CA1 area, even though the effect appears to be modest and should be confirmed in future experiments.

Given the importance of $\mathrm{Ca}^{2}$ influx for the initiation of most forms of LTP and short -term potentiation, we also examined the effects of mitragynine on $\mathrm{Ca}^{2}$ channels in cultured primary neurons from E16-E18 rats. Fluorescent imaging techniques using calcium-sensitive indicators such as Fura-2 are able to monitor changes in postsynaptic calcium levels, such as those seen during LTP induction. Application of glutamate $(2 \mu \mathrm{M})$ was found to enhance $\mathrm{Ca}^{2+}$ influx in cultured primary neurons (see Figure 5(a)). However, pre-treatment with mitragynine at concentration of 10 , 30 , and $100 \mu \mathrm{M}$ potently inhibited $\mathrm{Ca}^{2+}$ influx upon glutamate stimulation, findings that corroborate previous research. For example, Matsumoto et al. (2005) showed that mitragynine blocks $\mathrm{T}$ - and L-type $\mathrm{Ca}^{2+}$ channel currents and reduces $\mathrm{KCl}$-induced $\mathrm{Ca}^{2+}$ influx in neuroblastoma cells. Based on these results and on our observations of inhibition of glutamate-elicited $\mathrm{Ca}^{2+}$ influx, one can speculate that changes in $\mathrm{Ca}^{2+}$ signaling underlie the impairments of hippocampal transmission and plasticity, as well as of memory acquisition, seen with mitragynine administration.

The induction of long-lasting, late-phase LTP (L-LTP) is subserved by persistently activated protein kinases and involves changes in gene expression and protein synthesis. Specifically, L-LTP causes activation of cAMP-dependent protein kinase (PKA) and extracellular signal-related protein kinase (ERK) (Impey et al., 1998). Subsequently, several transcription factors such as calcium responsive-element binding protein (CREB) are phosphorylated and activated for the transcription of downstream genes to mediate the changes in the structure and/or function of the synapses. One of the downstream genes is $B d n f$. Thus, we examined the effect of mitragynine on the expression of $B d n f$. However, the results of these experiments did not reveal significant changes in $B d n f$ expression induced by the drug. These observations suggest that alternations in $B d n f$ functioning are unlikely to mediate the behavioral and electrophysiological effects noted after mitragynine administration.
LTP induction results in $\mathrm{Ca}^{2+}$ influx, triggering intracellular biochemical cascades through activation of alpha calciumcalmodulin-dependent protein kinase II ( $\alpha$ CaMKII), a major synaptic protein related to learning and memory in animals and humans (Easton et al., 2013a; Leonard et al., 1999, Lisman et al., 2012). The activation of $\alpha$ CaMKII plays a major role in plasticity induction and maintenance, as well as in behavioral learning (Bliss and Collingridge, 1993) and psychoactive drug use and abuse (Müller et al., 2016). Reinforcing drugs, which induce neuronal activation and LTP, have been shown to enhance $\alpha$ CaMKII activity (Easton et al., 2013a,b; Schöpf et al., 2015). However, this activity increase may be brought about by enhanced mRNA expression and/or phosphorylation (Müller et al., 2016). The results of the present experiments found no changes in the expression of the $\alpha$ CaMKII gene in mitragynine-treated rats. Whether $\alpha$ CaMKII phopsphorylation was enhanced still has to be evaluated in future studies. It should also be noted that LTP and memory formation may well be induced in the absence of $\alpha$ CaMKII and its phosphorylation, though at reduced speed (Radwanska et al., 2011; Villers et al., 2014). From that, one may conclude that the inhibitory mitragynine effects on glutamate-induced $\mathrm{Ca}^{2+}$ influx may translate to LTP deficits not by changing the $\alpha$ CaMKII or brain-derived neurotrophic factor (BDNF) pathways, but by other, yet to be determined mechanisms. This may, for instance, be brought about by altering the modulatory monoaminergic inputs to the hippocampus originating from the mesencephalon (Yusoff et al., 2016).

In conclusion, our study demonstrates that mitragynine caused profound impairments in spatial memory acquisition and excitatory synaptic transmission in the hippocampal CA1 field, together with a moderate suppression of LTP. Further, mitragynine application resulted in an inhibition of $\mathrm{Ca}^{2+}$ influx into neurons, raising the possibility that changes in $\mathrm{Ca}^{2+}$ signaling is involved in the behavioral and synaptic impairments elicited by this drug. Thus, mitragynine, particularly at higher doses, has the potential to cause significant cognitive dysfunction and disruption of processing in the hippocampal formation of rodents.

\section{Authors' contributions}

ZH, FWS, MAE designed, performed research and analyzed data. ZH, FWS, KHL, MAE, CPM, and HCD wrote the article. All authors read and approved the manuscript.

\section{Declaration of conflicting interests}

The author(s) declared no potential conflicts of interest with respect to the research, authorship, and/or publication of this article.

\section{Funding}

The author(s) disclosed receipt of the following financial support for the research, authorship and/or publication of this article: This work was supported by the Higher Education Centre of Excellence special funding (304/CDADAH/4401009), the International Research Collaboration Fund (1002/CDADAH/910410).

\section{ORCID iDs}

Zurina Hassan (iD https://orcid.org/0000-0003-3205-4612 
Mohamad A Effendy (iD https://orcid.org/0000-0002-1642-1390

\section{References}

Ahmad K and Aziz Z (2012) Mitragyna speciosa use in the northern states of Malaysia: A cross-sectional study. J Ethnopharmacol 141: 446-450.

Apryani E, Hidayat MT, Moklas M, et al. (2010) Effects of mitragynine from Mitragyna speciosa Korth leaves on working memory. $J$ Ethnopharmacol 129: 357-360.

Bannerman DM, Niewoehner B, Lyon L, et al. (2008) NMDA receptor subunit NR2A is required for rapidly acquired spatial working memory but not incremental spatial reference memory. $J$ Neurosci 28: 3623-3630.

Beckett A, Shellard E and Tackie A (1965) The Mitragyna species of Asia - Part IV the alkaloids of the leaves of Mitragyna speciosa Korth isolation of mitragynine and speciofoline. Planta Med 13: 241-246.

Bennett M (2000) The concept of long-term potentiation of transmission at synapses. Prog Neurobiol 60: 109-137.

Bliss T and Collingridge G (1993) A synaptic model of memory: Longterm potentiation in the hippocampus. Nature 361: 31-39.

Burkill IH (1935) A Dictionary of the Economic Products of the Malay Peninsula. Vol. II. pp. 1480-1483.

Burkill IH and Haniff M (1930) Malay village medicine. The Garden's Bulletin Straits Settlement 6: 165-207.

Chittrakarn S, Sawangjaroen K, Prasettho S, et al. (2008) Inhibitory effects of kratom leaf extract (Mitragyna speciosa Korth) on the rat gastrointestinal tract. J Ethnopharmacol 116: 173-178.

Chuakul W, Temsiririkkul R, Saralamp P, et al. (1995) Thai Medical Plants: The National Wisdom. Bangkok, Thailand: Amarin Printing and Publishing Ltd.

Damodaran T, Tan BW, Liao P, et al. (2018) Clitoria ternatea L root extract ameliorated the cognitive and hippocampal long-term potentiation deficits induced by chronic cerebral hypoperfusion in the rat. J Ethnopharmacol 224: 381-390.

de Moraes NV, Moretti RA, Furr EB, et al. (2009) Determination of mitragynine in rat plasma by LC-MS/MS: Application to pharmacokinetics. J Chromatogr 877: 2593-2597.

de Souza Silva MA, Silva MA, Lenz B, et al. (2013) Neurokinin3 receptor as a target to predict and improve learning and memory in the aged organism. Proc Natl Acad Sci U S A 110: 15097-15102.

Dunlop J, Bowlby M, Peri R, et al. (2008) High-throughput electrophysiology: An emerging paradigm for ion-channel screening and physiology. Nat Rev Drug Discov 7: 358-368.

Easton AC, Lourdusamy A, Loth E, et al. (2013a) CAMK2A polymorphisms predict working memory performance in humans. Mol Psychiatry 18: 850-852.

Easton AC, Lucchesi W, Lourdusamy A, et al. (2013b) $\alpha$ CaMKII autophosphorylation controls the establishment of alcohol drinking behavior. Neuropsychopharmacology 38: 1636-1647.

Grewal KS (1932a) Observations on the pharmacology of mitragynine. $J$ Pharmacol Exp Ther 46: 251-271.

Grewal KS (1932b) The effect of mitragynine on man. Br J Med Psychol 12: $41-58$.

Hassan Z, Muzaimi M, Navaratnam V, et al. (2013) From Kratom to mitragynine and its derivatives: Physiological and behavioural effects related to use, abuse, and addiction. Neurosci Biobehav Rev 37: $138-151$

Hazim AI, Mustapha M and Mansor SM (2011) The effects on motor behaviour and short-term memory tasks in mice following an acute administration of Mitragyna speciosa alkaloid extract and mitragynine. J Med Plants Res 5: 5810-5817.

Ilmie MU, Mansor SM and Abdullah JM (2015) Behavioural and electrophysiological evidence of impaired learning and memory in male Sprague Dawley rats following subchronic exposure to standardized methanolic extract of Mitragyna speciosa Korth. Malays J Med Sci Special Issue: $45-51$.

Impey S, Obrietan K, Wong ST, et al. (1998) Cross talk between ERK and PKA is required for $\mathrm{Ca}^{2+}$ stimulation of CREB-dependent transcription and ERK nuclear translocation. Neuron 21: 869-883.

Ismail NI, Jayabalan N, Mansor SM, et al. (2017) Chronic mitragynine (kratom) enhances punishment resistance in natural reward seeking and impairs place learning in mice. Addict Biol 22: 967-976.

Izquierdo LA, Barros DM, Ardenghi PG, et al. (2000) Different hippocampal molecular requirements for short- and long-term retrieval of one-trial avoidance learning. Behav Brain Res 111: 93-98.

Jamil MF, Subki MF, Lan TM, et al. (2013) The effect of mitragynine on cAMP formation and mRNA expression of mu-opioid receptors mediated by chronic morphine treatment in $\mathrm{SK}-\mathrm{N}-\mathrm{SH}$ neuroblastoma cell. J Ethnopharmacol 148: 135-143.

Janchawee B, Keawpradub N, Chittrakarn S, et al. (2007) A high-performance liquid chromatographic method for determination of mitragynine in serum and its application to a pharmacokinetic study in rats. Biomed Chromatogr 21: 176-183.

Jansen KL and Prast CJ (1988) Ethnopharmacology of kratom and the Mitragyna alkaloids. J Ethnopharmacol 23: 115-119.

Kruegel AC, Gassaway MM, Kapoor A, et al. (2016) Synthetic and receptor signaling explorations of the mitragyna alkaloids: Mitragynine as an atypical molecular framework for opioid receptor modulators. J Am Chem Soc 138: 6754-6764.

Leon F, Habib E, Adkins JE, et al. (2009) Phytochemical characterization of the leaves of Mitragyna speciosa grown in USA. Nat Prod Commun 4: 907-910.

Leonard AS, Lim IA, Hemsworth DE, et al. (1999) Calcium/ calmodulin-dependent protein kinase II is associated with the N-methyl-D-aspartate receptor. Proc Natl Acad Sci U S A 96: 3239 3244.

Li Z, Wu C, Pei G, et al. (2001) Reversal of morphine-induced memory impairment in mice by withdrawal in Morris water maze: Possible involvement of cholinergic system. Pharmacol Biochem Behav 68: $507-513$.

Lisman J, Yasuda R, Raghavachari S (2012) Mechanisms of CaMKII action in long-term potentiation. Nat Rev Neurosci 13: 169-82.

Luu-The V, Paquet N, Calvo E, et al. (2005) Improved real-time RT-PCR method for high-throughput measurements using second derivative calculation and double correction. BioTechnique 38: 287-293.

Lynch MA (2004) Long-term potentiation and memory. Physiol Rev 84: $87-136$

Matsumoto K, Yamamoto LT, Watanabe K, et al. (2005) Inhibitory effect of mitragynine, an analgesic alkaloid from Thai herbal medicine, on neurogenic contraction of the vas deferens. Life Sci 78: 187-194.

Matsumoto K, Narita M, Muramatsu N, et al. (2014) Orally active opioid/ dual agonist MGM-16, a derivative of the indole alkaloid mitragynine, exhibits potent antiallodynic effect on neuropathic pain in mice. J Pharmacol Exp Ther 348: 383-392.

McNamara RK and Skelton RW (1992) Pharmacological dissociation between the spatial learning deficits produced by morphine and diazepam. Psychopharmacology 108: 147-152.

Müller CP, Quednow BB, Lourdusamy A, et al. (2016) CaM kinases: From memories to addiction. Trends Pharmacol Sci 37: 153-166.

Niewoehner B, Single FN, Hvalby Ø, et al. (2007) Impaired spatial working memory but spared spatial reference memory following functional loss of NMDA receptors in the dentate gyrus. Eur $J$ Neurosci 25: 837-846.

Orio L, Alexandru L, Cravotto G, et al. (2012) UAE, MAE, SFE-CO2 and classical methods for the extraction of Mitragyna speciosa leaves. Ultrason Sonochem 19: 591-595.

Parthasarathy S, Ramanathan S, Ismail S, et al. (2010) Determination of mitragynine in plasma with solid-phase extraction and rapid HPLC- 
UV analysis, and its application to a pharmacokinetic study in rat. Anal Bioanal Chem 397: 2023-2030.

Ponglux D, Wongseripipatana S, Takayama H, et al. (1994) A new indole alkaloid, $7 \alpha$-Hydroxy-7H-mitragynine, from Mitragyna speciosa in Thailand. Planta Med 60: 580-581.

Radwanska K, Medvedev NI, Pereira GS, et al. (2011) Mechanism for long-term memory formation when synaptic strengthening is impaired. Proc Natl Acad Sci U S A 108: 18471-18475.

Ramanathan S, Parthasarathy S, Murugaiyah V, et al. (2015) Understanding the physicochemical properties of mitragynine, a principal alkaloid of mitragyna speciosa, for preclinical evaluation. Molecules 20: 4915-4927.

Said I, Chun N and Houghton P (1991) Ursolic acid from Mitragyna speciosa. Planta Med 57: 398-398.

Schöpf I, Easton AC, Solati J, et al. (2015) aCaMKII autophosphorylation mediates neuronal activation in the hippocampal dentate gyrus after alcohol and cocaine in mice. Neurosci Lett 591: 65-68.

Senik MH, Mansor SM, Rammes G, et al. (2012) Mitragyna speciosa Korth standardized methanol extract induced short-term potentiation of CA1 subfield in rat hippocampal slices. J Med Plants Res 6: 1234-1243.

Shellard EJ (1974) The alkaloids of Mitragyna with special reference to those of Mitragyna speciosa Korth. Bull Narc 26: 41-55.

Shellard EJ (1989) Ethnopharmacology of kratom and the Mitragyna alkaloids. J Ethnopharmacol 25: 123-124.

Shellard EJ, Houghton PJ and Resha M (1978a) The Mitragyna species of Asia: Part XXXI: The alkaloids of Mitragyna speciosa Korth from Thailand. Planta Med 34 (5): 26-36.

Shellard EJ, Houghton PJ and Resha M (1978b) The Mitragyna species of Asia: Part XXXII: The distribution of alkaloids in young plants of Mitragyna speciosa Korth grown from seed obtained from Thailand. Planta Med 34: 253-263.

Simon W, Hapfelmeier G, Kochs E, et al. (2001) Isoflurane blocks synaptic plasticity in the mouse hippocampus. Anesthesiology 94: 1058-1065.

Singh D, Müller CP and Vicknasingam BK (2014) Kratom (Mitragyna speciosa) dependence, withdrawal symptoms and craving in regular users. Drug Alcohol Depend 139: 132-137.

Suhaimi FW, Yusoff NH, Hassan R, et al. (2016) Neurobiology of Kratom and its main alkaloid mitragynine. Brain Res Bull 126: 29-40.

Suwanlert S (1975) A study of kratom eaters in Thailand. Bull Narc 27 (3): 21-27.

Takayama H, Kurihara M, Kitajima M, et al. (1998) New indole alkaloids from the leaves of Malaysian Mitragyna speciosa. Tetrahedron 54: 8433-8440.
Tanguay P (2011) Kratom in Thailand. Legis Reform Drug Pol 13: 1-16. Taufik Hidayat M, Apryani E, Nabishah BM, et al. (2010) Determination of mitragynine bound opioid receptors. Adv Med Dent Sci 3: 65-70.

Thomson AM (2000) Molecular frequency filters at central synapses. Prog in Neurobio 62(2): 159-196.

Utar Z, Majid MI, Adenan MI, et al. (2011) Mitragynine inhibits the COX-2 mRNA expression and prostaglandin E2 production induced by lipopolysaccharide in RAW264 7 macrophage cells. J Ethnopharmacol 136: 75-82.

Vicknasingam B, Narayanan S, Beng GT, et al. (2010) The informal use of ketum (Mitragyna speciosa) for opioid withdrawal in the northern states of peninsular Malaysia and implications for drug substitution therapy. Int J Drug Pol 21: 283-288.

Villers A, Giese KP and Ris L (2014) Long-term potentiation can be induced in the CA1 region of hippocampus in the absence of $\alpha$ CaMKII T286-autophosphorylation. Learn Mem 21: 616-626.

Vorhees CV and Williams MT (2006) Morris water maze: Procedures for assessing spatial and related forms of learning and memory. Nat Protoc 1: 848-858.

Watanabe K, Yano S, Horie S, et al. (1997) Inhibitory effect of mitragynine, an alkaloid with analgesic effect from Thai medicinal plant Mitragyna speciosa, on electrically stimulated contraction of isolated guinea-pig ileum through the opioid receptor. Life Sci 60: 933-942.

Wray I (1907a) Biak: An opium substitute. Journal of the Federated Malay States Museums 53-56.

Wray I (1907b) Notes on the anti-opium remedy. Pharm J 78: 453.

Yusoff NH, Suhaimi FW, Vadivelu RK, et al. (2016) Abuse potential and adverse cognitive effects of mitragynine (kratom). Addict Biol 21: 98-110.

Yusoff NHM, Mansor SM, Muller CP, et al. (2018) Baclofen blocks the acquisition and expression of mitragynine-induced conditioned place preference in rats. Behav Brain Res 345: 65-71.

Zheng XG, Li XW, Yang XY, et al. (2002) Effects of scopolamine and physostigmine on acquisition of morphine-treated rats in Morris water maze performance. Acta Pharmacologica Sinica 23: 477-480.

Zhu F, Yan CX, Zhao Y, et al. (2011) Effects of pre-training morphine on spatial memory acquisition and retrieval in mice. Physiol Behav 104: 754-760.

Zucker RS (1989) Short-term synaptic plasticity. Annu Rev Neurosci 12: 13-31. 